October 27, 2003

hep-th/0310221

MIT-CTP-3426

SLAC-PUB-10211

SU-ITP-3/29

NSF-KITP-03-87

\title{
Scalar Speed Limits and Cosmology: Acceleration from D-cceleration
}

\author{
Eva Silverstein ${ }^{1}$ and David Tong ${ }^{2}$ \\ ${ }^{1} S L A C$ and Department of Physics, Stanford University, \\ Stanford, CA 94309/94305, U.S.A. \\ evas@slac.stanford.edu \\ ${ }^{2}$ Center for Theoretical Physics, Massachusetts Institute of Technology, \\ Cambridge, MA 02139, U.S.A. \\ dtong@mit.edu
}

\begin{abstract}
Causality on the gravity side of the AdS/CFT correspondence restricts motion on the moduli space of the $\mathcal{N}=4$ super Yang Mills theory by imposing a speed limit on how fast the scalar field may roll. This effect can be traced to higher derivative operators arising from integrating out light degrees of freedom near the origin. In the strong coupling limit of the theory, the dynamics is well approximated by the Dirac-Born-Infeld Lagrangian for a probe D3-brane moving toward the horizon of the AdS Poincare patch, combined with an estimate of the (ultimately suppressed) rate of particle and string production in the system. We analyze the motion of a rolling scalar field explicitly in the strong coupling regime of the field theory, and extend the analysis to cosmological systems obtained by coupling this type of field theory to four dimensional gravity. This leads to a mechanism for slow roll inflation for a massive scalar at subPlanckian VEV without need for a flat potential (realizing a version of k-inflation in a microphysical framework). It also leads to a variety of novel FRW cosmologies, some of which are related to those obtained with tachyon matter.
\end{abstract}

Stanford Linear Accelerator Center, Stanford University, Stanford, CA 94309

Work supported in part by the Department of Energy contract DE-AC03-76SF00515. 


\section{Introduction}

It is now almost 100 years since Einstein introduced the concept of a universal speed limit for all physical systems propagating in spacetime. However, in general, motion on the configuration space of a physical system is not constrained to obey a speed limit. For example, the vacuum expectation values (VEVs) of light scalar fields naturally move in an internal moduli space where motion is uninhibited; within classical, relativistic field theory there is no restriction on the rate of change of the VEV. The purpose of this paper is to show that this situation does not necessarily continue to hold in the quantum theory. We exhibit situations where the strong coupling dynamics do impose a speed limit on an internal moduli space, and examine applications to cosmology, including a mechanism for slow roll inflation.

We focus on the $\mathcal{N}=4$ supersymmetric Yang Mills (SYM) theory. Although the (supersymmetry-protected) metric on the moduli space is flat, the quantum induced speed limit ensures that a rolling scalar field slows down as it approaches the origin. This fact can be seen immediately from the gravity side of the AdS/CFT correspondence, where the process corresponds to a D3-brane domain wall in $A d S_{5}$ moving toward the horizon. The familiar causal speed-limit in the bulk translates into a speed limit on moduli space which becomes more pronounced as the brane approaches the origin. Indeed, from the perspective of a boundary observer, the probe brane takes an infinite time to cross the horizon (though as we will discuss, the probe approximation breaks down due to back reaction at very late times). This speed limit on the moduli space, arising from causality in the bulk, was first stressed by Kabat and Lifschytz [1].

On the field theory side of the correspondence, this result reflects the breakdown of the moduli space $\sigma$-model approximation as a scalar field approaches a locus with new light degrees of freedom where higher derivative terms become important. In AdS/CFT dual pairs, we can use the gravity side of the correspondence to determine the net effect of these higher-derivative corrections where they are summed into a Dirac-Born-Infeld (DBI) action. The resulting dynamics is dramatically different from naive expectation based on the supersymmetric moduli space metric.

In this paper, we will study the dynamics explicitly, both in quantum field theory in its own right and in the cosmological context arising from quantum field theory coupled to four dimensional gravity. We use the dual picture of $D 3$-branes and anti- $D 3$-branes moving in an $A d S$-like throat and we exhibit late time solutions describing the physics as the scalar field approaches the origin. Among our results, we find that the slowing 
down of the scalar field (relative to the behavior predicted by the two derivative action) can lead to new regimes exhibiting inflationary behavior.

The idea of obtaining inflation from $\overline{D 3}$ and $D 3$ branes in a warped throat was studied recently in 2]. In particular, the authors observe that one can gain extra control from the warping, but some obstacles to obtaining explicit inflationary models in string compactifications were identified. Our results here concern the effects of the crucial higher derivative terms in the effective action and allow us to probe a different regime from that studied in 2] which may help address the challenges of [2]. In particular the slow motion of the scalar field, enforced by higher derivative terms, leads to a new mechanism for slow roll inflation. The inflation occurs in a sub-Planckian regime for the scalar field, and is robust against curvature corrections such as the conformal coupling of the scalar to the four dimensional scalar curvature.

A scalar field mass is required to obtain our simplest inflationary solution. The required mass scale is not finely tuned in ordinary four dimensional effective field theory terms, which provides an interesting distinction from the usual inflationary models. In the case of the $\mathcal{N}=4$ super Yang Mills theory coupled to gravity, we propose some effective field theory couplings to other sectors generating such a mass while leaving intact the form of the crucial kinetic corrections to the moduli space approximation of the field theory. However, we should note that the couplings of Kaluza Klein modes in the corresponding brane throat to other sectors may be important and may require tuned coefficients to avoid destabilization of the throat; this is a little understood aspect of current compactification technology. Because of the plethora of independent ingredients (and types of domain wall branes) available in string compactifications, we expect the combination of approximate AdS-metric induced kinetic terms and scalar masses generated from other couplings is likely to be available in some set of examples. It remains the main weakness of our results however that we will not exhibit an explicit example here. The other field theoretic and cosmological phases we will exhibit are not tied to this subtlety.

Our results have further relations to previous work. The use of higher derivative terms to change scalar field dynamics in ways interesting for cosmology has been dubbed "k-inflation" [3] or "k-essence" 4] . A particularly well-studied example occurs in the effective field theory describing the decay of branes and antibranes leading to "tachyon matter" [5, 6, 7] and the associated cosmology [8, 9]. While our system shares many features with the rolling tachyon story, including the existence of a pressureless dust equation of state, it also differs in several ways important for cosmology. In par- 
ticular, we shall argue that particle and string production is suppressed in our system and the dynamics is governed to good approximation by our equations of motion. For a few examples of previous works studying the application of rolling moduli to string cosmology see [10, 11, 12] and the review [13]. The crucial physics of our model is ultimately extracted from the dynamics of D-branes; there are of course many interesting investigations in this area, for example [14, 15, 16].

The manner in which higher derivative terms can drastically affect the dynamics of a system raises many questions concerning potential applications to real models of cosmology as well as more theoretical issues. Among the latter set is the question of whether or not motion toward other finite distance singularities in field theory and string theory moduli spaces (such as the conifold singularity) also exhibits similar slowing down effects for some regime of the couplings. Noticeably, recent studies of the flop transition, which explore the effects of the states which become light at the singularity, suggest that the rolling scalar field does indeed become stuck in the region of the singularity [17, 18].

The paper is organized as follows. We start in Section 2 by describing our basic set-up, stressing the appearance of a speed limit on the moduli space. In Section 3 we study the consequences of this speed limit for the dynamics in the global conformal field theory. We then couple our system to gravity in Section 4. We take particular care to describe the possible deformations of the system arising from other sectors in a string compactification, including the generation of a potential on the moduli space and the effects this has on the AdS geometry seen by the probe brane. In Section 5 we study cosmologies arising from our low-energy effective actions. In Section 6 , we check that our solutions are not destabilised by perturbations or particle production.

\section{The Basic Setup}

Typically studies of scalar field dynamics consider an effective field theory Lagrangian containing a kinetic term (up to two derivatives) together with a potential energy on the space of scalar fields:

$$
S_{\text {two deriv }}=\int d^{4} x \sqrt{g}\left(G_{i j}(\phi) g^{\mu \nu} \partial_{\mu} \phi^{i} \partial_{\nu} \phi^{j}-V(\phi)\right)
$$

In supersymmetric situations, the moduli space metric $G_{i j}$ and the form of the potential energy $V$ are highly constrained, and much of the recent work on such quantum field 
theories has focused on determining these quantities and the BPS spectrum of states exactly.

It is well known however that the moduli space approximation (in which the physics is governed by the action (2.1) along flat directions of the potential $V(\phi)$ ) can break down due to the presence of new light degrees of freedom arising on special loci of the space of scalar field VEVs.

In the $U(N) \mathcal{N}=4 \mathrm{SYM}$ theory, one has scalar fields in the adjoint representation of the gauge group which can be represented by $N \times N$ matrices $\Phi$. The moduli space of the field theory is parameterized by diagonal (commuting) matrices, with eigenvalues $\phi_{1}, \ldots, \phi_{N}$ whose moduli space metric, including quantum corrections, is flat. Away from the origin $\phi_{i}=0$ of this moduli space, the low energy gauge symmetry is generically $U(1)^{N}$ and off diagonal matrix elements of the scalar fields, fermions, and gauge bosons obtain masses. We shall refer to these modes collectively as "W bosons". In particular, we will consider a configuration in which $\left\langle\phi_{1}\right\rangle \equiv\langle\phi\rangle \neq 0$ but all the other $\phi_{i}, i \neq 1$ have vanishing VEVs. This means that the theory has a low energy unbroken gauge symmetry $U(N-1) \times U(1)$ with massive $\mathrm{W}$ bosons in the $(\mathbf{N}-\mathbf{1},+) \oplus(\overline{\mathbf{N}-\mathbf{1}},-)$ representation. The $\mathrm{W}$ bosons are BPS protected states with mass

$$
m_{W}=\phi
$$

We will work expanding about the large $N$ limit of the theory, in which the natural parameters in the field theory are the rank $N$ and the 't Hooft coupling $\lambda=g_{Y M}^{2} N$ in terms of the Yang Mills coupling $g_{Y M}$.

\subsection{The System at Weak Coupling}

At weak coupling in the field theory, the effective action for $\phi$ gets contributions from virtual $\mathrm{W}$ bosons. For large $\langle\phi\rangle$, these contributions scale like powers of $\lambda \dot{\phi}^{2} / \phi^{4}$ in the planar limit (see [19] for a comprehensive discussion of these higher derivative corrections). If we send the scalar field rolling toward the origin from a finite point on the Coulomb branch, the classical action (2.1) would predict a constant velocity $\dot{\phi}=v_{0}$, but the form of the corrections just noted shows that the action (2.1) becomes unreliable at the distance

$$
\phi^{2}=\sqrt{\lambda} v_{0}
$$


The question then arises about how to take into account these corrections. However, in the weak coupling regime, the point is moot as we have another issue to confont before we get this close to the origin. Since the $\mathrm{W}$ bosons become light, they may be produced on shell during the evolution. The time dependent mass leads to particle production controlled by the parameter $\dot{m}_{W} / m_{W}^{2}$, which therefore becomes important at the distance,

$$
\phi^{2}=v_{0}
$$

For $\lambda \ll 1$, we first reach the production point (2.4).

Naively extrapolating the above perturbative results to the strong coupling regime, we reach the point (2.3) first and therefore expect that the dynamics will be governed by the effective action for $\phi$ taking into account the $\lambda \dot{\phi}^{2} / \phi^{4}$ corrections arising from virtual $\mathrm{W}$ bosons. In the following section we shall confirm this expectation and show that the scale (2.3) is where the speed limit on the moduli space first becomes apparent.

\subsection{The System at Strong Coupling and the Speed Limit}

At strong coupling $\lambda \gg 1$, the effective description of the theory is via gravity and string theory using the AdS/CFT correspondence [20]. A point on the Coulomb branch is described on the gravity side in the Poincare patch via a D3-brane at fixed radial position $r$ in the metric

$$
d s^{2}=\frac{r^{2}}{R^{2}}\left(-d t^{2}+d x^{2}\right)+\frac{R^{2}}{r^{2}} d r^{2}
$$

and at a point on the $S^{5}$. The field theory coordinate $\phi$ on the Coulomb branch translates into $r / \alpha^{\prime}$ in the gravity variables. We further have the relations $R=\left(g_{s} N\right)^{1 / 4} \sqrt{\alpha^{\prime}}$ and $g_{Y M}^{2}=4 \pi g_{s}$.

As in other applications of AdS/CFT [20] and Randall-Sundrum [21], the warp factor in (2.5) plays an important role in understanding the energy spectrum of the system. The string mass scale at position $r$ is related to that at position $r^{\prime}$ by the ratio of warp factors $r / r^{\prime}$. The open string oscillator modes on the brane at position $r=\langle\phi\rangle \alpha^{\prime}$ have masses of order

$$
m_{s}(\langle\phi\rangle)=\frac{\phi}{\lambda^{1 / 4}}
$$

As in the Randall Sundrum scenario, the effective cutoff for modes on the brane is at this warped string energy scale (2.6) rather than the UV string scale $1 / \sqrt{\alpha^{\prime}}$. 
The $\mathrm{W}$ bosons are strings stretched from the brane to the horizon at $r=0$. Although this is an infinite proper distance for the string to stretch, the warp factor $r / R$ reduces the effective tension of the string enough to produce a finite total mass $m_{W}=\phi$ in accord with the BPS formula (2.2).

We would now like to highlight the simple feature of our system which lies behind most of our detailed results to follow. It is immediately clear from (2.5) that, for a boundary observer using coordinates $(t, x)$, a probe brane takes infinite time to reach the origin of the Coulomb branch at $r=0$. (In our solutions it will turn out that the proper time for the probe to fall to the origin will be finite.) The radial velocity of the D3-brane in the AdS space is limited by the speed of light which, translated in field theory variables, becomes

$$
\dot{\phi} \leq \dot{\phi}_{c}=\frac{\phi^{2}}{\sqrt{\lambda}}
$$

This restriction was noted previously by Kabat and Lifschytz [1], who discussed some interesting aspects of the phenomenon. Note that the probe brane takes infinite time despite the fact that the moduli space metric in (2.1) for the $\mathcal{N}=4$ SYM theory is uncorrected quantum mechanically; the distance to the origin in the field theory moduli space metric is finite for any value of the coupling. As discussed above, the fact that it takes an infinite time to reach the origin arises from crucial corrections to the moduli space approximation. These corrections apply to any physical process in which the scalar rolls toward the origin of the Coulomb branch.

Note that the $\lambda \dot{\phi}^{2} / \phi^{4}$ corrections, while they are higher derivative corrections, are not suppressed by powers of the Planck mass; as we discussed above they are suppressed only by the $\mathrm{W}$ boson mass $\phi$. Let us compare this situation to a brane in flat space: such an object has a Lagrangian proportional to $\sqrt{1-v^{2}}$ where $v$ is the proper velocity of the brane. Written in terms of canonically scaled brane fields $\phi$, this becomes (for a D3-brane) $\sqrt{1-\dot{\phi}^{2} \alpha^{\prime 2}}$ which expands to a series of higher derivative terms which are suppressed by powers of the string mass $m_{s}$. In the global limit $m_{s} \rightarrow \infty$, these corrections die and the motion on $\phi$ space is unconstrained. It is the warp factor in (2.5) that produces higher derivative effects that are crucial at the field theory level, as we will see in detail in our analysis. 


\section{The Global CFT}

The corrections to the moduli space approximation, and the resulting dynamics, can be understood rather explicitly in a controlled analysis on the gravity side in which the rolling scalar VEV is modelled by a moving D3-brane probe in $A d S_{5}$. In this section we examine the resulting dynamics of the scalar field.

\subsection{Effective Action and Approximation Scheme}

On the gravity side, the effective Lagrangian appropriate for a probe D-brane at arbitrary velocity (less than or equal to the speed of light) but low proper acceleration is the Dirac-Born-Infeld Lagrangian. This Lagrangian describes the effects of virtual open strings at the planar level; it includes the effects of the background geometry and field strengths but does not include production of on shell closed strings or $\mathrm{W}$ bosons or loops of closed strings. We will analyze the motion of the 3-brane starting from this Lagrangian, and check for self consistency of the resulting solutions. This requires checking that the proper acceleration is small and taking the string coupling to be small so that the DBI action is a good approximation to the effective action of the probe. It further requires determining the range of parameters and times for which the energy in the probe does not back react significantly on the geometry, so that the probe approximation remains valid. Finally, we must also check that fluctuations about the solution, density perturbations, and particle and string production are not too large. In Section 4 we will consider generalizations of this system to include coupling to four dimensional gravity and other sectors, including effects of a cutoff throat in the IR; we will take into account new contributions to the action at the level of its most relevant terms at low energy.

In this section we start by writing down the effective action applicable to the pure conformal field theory (CFT) without gravity (infinite $A d S$ space). The DBI action for a probe D3-brane moving in $\mathrm{AdS}_{5} \times \mathrm{S}^{5}$ can be found in [20] and we will use their conventions. We concentrate only on radial fluctuations, and set the field strength on the brane to vanish. Working in field theory variables $\phi \equiv r / \alpha^{\prime}$, we have

$$
S=-\frac{1}{g_{Y M}^{2}} \int d t d^{3} x f(\phi)^{-1}\left[-\operatorname{det}\left(\eta_{\mu \nu}+f(\phi) \partial_{\mu} \phi \partial_{\nu} \phi\right)^{1 / 2}-1\right]
$$

where for now we take the background brane metric to be flat $\eta_{\mu \nu}=\operatorname{diag}(-1,1,1,1)$, and $f(\phi)$ is the harmonic function

$$
f(\phi)=\frac{\lambda}{\phi^{4}}
$$


Expanding the action out in derivatives leads to a canonical kinetic term for $\phi \equiv r / \alpha^{\prime}$ and a series of higher order derivative interactions. The potential cancels out, reflecting the BPS nature of the D3-brane. In fact, for a single excited scalar field $\phi$, this form of the higher derivative action can be shown to hold even at weak coupling [22] without recourse to a dual gravitational description: it is fixed by the requirement of a non-linearly realised conformal invariance, together with known non-renormalisation theorems.

So in general the dynamics of $\phi$ in our strongly coupled CFT differs in two important ways from a naive scalar field system governed by (2.1). Firstly, the kinetic term is corrected to that in (3.8); as discussed above we will find that the proper velocity approaches the speed of light so that the quantity in the square root approaches zero, resulting in a system far away from the regime where the two derivative action suffices. Secondly, there is no potential $V(\phi)$. In later sections we shall remedy the latter issue by generalizing to systems in which potentials are generated, both by anti-branes and by considering a field theory coupled to other sectors. The speed limit will remain a crucial ingredient in our generalizations.

\subsection{Dynamics}

Let us now study more explicitly the approach of the $\mathcal{N}=4$ SYM theory to the origin of its Coulomb branch, using the action (3.8). Since we are interested only in the time dependence of the solution, we shall ignore the spatial derivatives, leaving us with the action,

$$
S=-\frac{N}{\lambda^{2}} \int d^{4} x \phi^{4}\left(\sqrt{1-\lambda \dot{\phi}^{2} / \phi^{4}}-1\right)
$$

To determine the late time behaviour ${ }^{1}$ of this system, we first compute the conserved energy density $E$, given by (up to a factor of $1 / g_{Y M}^{2}$ )

$$
E=\frac{1}{\lambda} \phi^{4}\left(\frac{1}{\sqrt{1-\lambda \dot{\phi}^{2} / \phi^{4}}}-1\right) \approx \frac{1}{2} \dot{\phi}^{2}+\frac{1}{8} \frac{\lambda \dot{\phi}^{4}}{\phi^{4}}+\ldots
$$

where, in the second line, we have expanded around large $\phi$. The first term is the canonical kinetic energy for a rolling scalar field, with subsequent terms becoming important at small $\phi$, capturing the effect of virtual $\mathrm{W}$ bosons.

\footnotetext{
${ }^{1}$ Readers who prefer $u=1$ units may make the substitution in all expressions of this type.
} 
As mentioned above, the Born-Infeld action is valid for arbitrarily high velocities $\dot{\phi}$ but only for small proper accelaration $a$,

$$
a \sqrt{\alpha^{\prime}}=\frac{\lambda^{1 / 4}}{\phi} \frac{d}{d t}\left(\frac{\sqrt{\lambda} \dot{\phi}}{\phi^{2}}\right)
$$

So in order for our analysis to be self-consistent, we must check that the proper acceleration is much smaller than string scale in our solution. Inverting (3.11) to solve for $\dot{\phi}$, and subsequently taking the time derivative, it is simple to see that we can trust the DBI analysis when we are in the strong coupling regime $\lambda \gg 1$.

Integrating once, the trajectory of the scalar $\phi$ is given by,

$$
t-t_{0}=\frac{1}{\sqrt{E}} \int_{\phi_{0}}^{\phi} d \varphi \frac{1}{\varphi^{2}} \frac{\lambda E+\varphi^{4}}{\sqrt{\lambda E+2 \varphi^{4}}}
$$

We are interested in the dynamics of the scalar field after we hit the critical point on moduli space defined by (2.3). Using the classical expectation $E \sim v_{0}^{2}$, this means we are interested in the regime

$$
\phi^{4} \ll \lambda E
$$

Here we find the late time behaviour,

$$
\phi(t) \rightarrow \frac{\sqrt{\lambda}}{t}
$$

saturating the speed limit (2.7). We therefore find that from the perspective of the field theory observer, Xeno is vindicated and the scalar field takes an infinite time to reach the origin as advertised.

\subsubsection{Background Check}

The above calculation treated the D3-brane as a probe and is valid only when the back reaction can be neglected. Since the brane travels at almost the speed of light at late times on the gravity side, it carries a lot of energy and we must determine the conditions under which its back reaction does not destabilize the AdS background. ${ }^{2}$ The gravitational field surrounding a highly boosted object in locally flat space is given by the Aichelburg-Sexl metric [23, which in our case of codimension 6 is given by

$$
h_{--} \sim \frac{l_{s}^{8} g_{s} E_{p}}{r_{\perp}^{3}} \delta\left(x^{-}\right)
$$

\footnotetext{
${ }^{2}$ This constraint was obtained via discussions with M. Fabinger.
} 
Here $E_{p}=E(R / r)^{4}$ is the proper energy density of the probe (related to the Poincare observer's energy density $E$ by the appropriate powers of the warp factor $r / R), r_{\perp}$ is the distance from the probe in the transverse dimensions, and $x^{-}$is the light cone coordinate with respect to which the brane trajectory is localized in locally flat coordinates. If we insist that the corresponding curvature $\mathcal{R}_{10} \sim h_{--} / r_{\perp}^{2}$ smeared over a string scale distance across the brane be smaller than the ambient AdS curvature $1 / R^{2}$ at a distance $R$ from the probe, this gives the condition

$$
E<\frac{\phi^{4}}{\lambda^{1 / 4} g_{s}}
$$

If we impose this condition, we still obtain a window

$$
\lambda^{1 / 4} g_{s} E<\phi^{4}<\lambda E
$$

in which our back reaction constraint (3.15) intersects with our regime (3.12) of limiting speed.

This constraint (3.15) may be too strong, since the novel behavior we found for $\phi$ based on the DBI action depends only on the AdS geometry. We can estimate the back reaction of our probe on the AdS geometry as follows. The probe forms a domain wall of energy density $E_{p}$ in the five dimensional gravity theory obtained by dimensional reduction on the $S^{5}$. Such a wall will jump the warp factor accross it. By dimensional analysis, this gives the relation

$$
\frac{1}{R^{\prime}}-\frac{1}{R} \sim E_{p} l_{5}^{3}
$$

in terms of the five dimensional Planck length $l_{5}$, where $R^{\prime}$ is the curvature radius on the IR side of the domain wall. Using $l_{5}^{3} \sim l_{10}^{8} / R^{5}=l_{s}^{8} g_{s}^{2} / R^{5}$, the condition that the jump in warp factor is smaller than the original AdS warp factor is

$$
E<\frac{\phi^{4}}{g_{s}}
$$

Again, this leads to a window at strong coupling in which the rolling scalar field saturates its speed limit (3.13) in the regime

$$
g_{s} E<\phi^{4}<\lambda E
$$

Similar back reaction criteria apply to all the cases discussed in the paper, and we will derive a related bound in our interesting inflationary phase. It would be interesting to explore what happens when this condition is violated. We will discuss the other consistency conditions for our background (including ruling out significant back reaction from particle production) in Section 6 . 


\subsection{Antibranes}

Here we introduce the first of our generalizations: anti-D3-branes moving in the AdS background (2.5). The action for the $\overline{D 3}$-brane probe in $A d S_{5}$ differs from the $D 3$-brane case (3.10) merely by a change of sign in the final term,

$$
S=-\frac{N}{\lambda^{2}} \int d^{4} x \phi^{4}\left(\sqrt{1-\lambda \dot{\phi}^{2} / \phi^{4}}+1\right)
$$

Upon expanding the square-root in powers of $\lambda \dot{\phi}^{2} / \phi^{4}$, this gives rise to a potential which is quartic in $\phi$. There is no quadratic $m^{2} \phi^{2}$ term. This may be at first sight surprising since the system has broken supersymmetry in the presence of the antibrane, and loops of open strings which probe the susy breaking are included at the level of the action (3.18). However this result is to be expected using simple Randall-Sundrum ideas. The local string scale at the position of the brane is $\phi / \lambda^{1 / 4}(2.6)$. Similarly all hard masses are warped down by a factor of $r / R=\phi \sqrt{\alpha^{\prime}} / \lambda^{1 / 4}$ at position $\phi$ in the throat. Applying this to the susy breaking scale $m_{S U S Y}$, a potential $V \sim m_{S U S Y}^{2} \phi^{2}$ really scales like $\phi^{4}$. Said differently, there is no independent scale in the CFT on the Coulomb branch beyond the position $\phi$.

The $\phi^{4}$ potential implies that the $\overline{D 3}$-brane experiences a force towards the horizon at $r=0$. Nonetheless, it is a simple matter to check that the late time dynamics is dominated by the speed limit and is identical to that of the D3-brane (3.13).

In some sense, the $\overline{D 3}$-brane probe in $A d S$ space can be thought of as a strong coupling limit of the usual tachyon matter system. Indeed, we shall see in Section 5 that the resulting dynamics bears some similarity to the results that have been obtained for the much-studied open string tachyon decay in the weakly coupled braneantibrane system (see 24] for recent review of this system). However, there are some differences which we stress here. At weak coupling, the $\bar{D} 3$-brane system has tachyons from strings stretching between the antibrane and the $N-1$ D3-branes described by the AdS throat. However, at strong coupling $\lambda \gg 1$, it is easy to see from (2.2) and (2.6) that this mode is not tachyonic. The negative contribution to the would-be tachyon mass squared, scaling like the (warped) string scale $m_{s}(\langle\phi\rangle)^{2}$, is dwarfed by the

positive contribution $m_{W}^{2}$ to the mass squared from the stretching of the string from the antibrane to the AdS horizon. Thus the brane-antibrane system at strong 't Hooft coupling (obtained by taking many branes and a single antibrane) has no tachyon. The system is unstable - as we will discuss in detail the antibrane falls towards the horizon. However this evolution is not condensation of a brane-antibrane tachyon since none 
exists; as in 25] the annihilation of the antibrane against the unit of D3-brane charge encoded in the RR flux is a nonperturbative effect.

\section{Coupling to Gravity}

In both the brane and antibrane cases, we will be interested in two further important generalizations. One is coupling to four dimensional gravity, as well as to other sectors suppressed by higher dimension operators that may arise in the corresponding string compactifications. The other is a generalization to a throat which is capped off in the region corresponding to the IR behavior of the field theory - i.e. the system with a mass gap. In a string theoretic setup, these effects can be achieved by gluing an AdS-throat like solution onto a compactification geometry which acts as a UV-brane as in [26, 27].

In this section we shall discuss the effective actions which result from these generalizations, focusing on the relative strength of corrections to the potential energy and DBI kinetic energy terms in the effective action. Our motivation for considering these theories is the application of the speed limit mechanism to cosmology. We will postpone a full discussion of this until Section 5. To avoid undue suspense, let us here summarize some of the important results.

Because our matter sector itself slows the scalar field $\phi$ in its progress toward the origin (in the field theory variables), the conditions for slow roll inflation are modified in a useful way. In a situation with a strong enough additional potential energy for $\phi$ (which may arise for example from a setup with a brane or antibrane in a cutoff AdS throat coupled to other sectors) we will indeed find a phase of slow roll inflation. This can be obtained at subPlanckian VEV for $\phi$, with the slow roll provided by the DBI kinetic term corrections rather than from the usual gravitational damping or from an unnaturally flat potential. This allows us to evade some of the problems discussed in [2]. While we have not yet produced a complete model of real-world inflation from this mechanism, it seems a very promising ingredient.

More generally, we will find some familiar behaviors for the FRW scale factor $a(t)$ but arising in unusual ways from our matter sector. For example, as in "tachyon matter" we will find a dust equation of state for motion of a D3-brane toward the origin of the Coulomb branch, though in our case production of massive matter is suppressed. With

other potentials, we also find a novel steady state late-time behaviour for the universe 
that does not involve fine tuning of initial conditions.

The motion of the moduli themselves is an important aspect of string cosmology, and the basic result of the previous sections leads to interesting novelties in this area. In particular, if the moduli field $\phi$ corresponding to the motion of the threebrane heads toward the origin of the Coulomb branch, it gets slowed down and, at least in the probe limit, takes an infinite time to reach the origin. This is radically different from the naive treatment based on the moduli space metric in which it would shoot past the origin without pausing. Furthermore, if the scalar relaxes into a potential well surrounding the origin, it does not oscillate around the minimum in the same manner as moduli treated with the usual action (2.1). However in the cases when the $\phi$ sector with all the DBI corrections leads to a dust equation of state, it can cause similar problems to the usual moduli problem.

\subsection{CFT Coupled to Gravity}

We firstly ask what becomes of the low-energy effective action (3.8) when the gauge theory is coupled to gravity by introducing a dynamical background metric $g_{\mu \nu}$. At the same time, we add a potential term $V(\phi)$ to the action that may arise when the system is coupled to four dimensional gravity and other sectors involved in a full string compactification. A simple four dimensional covariantization of (3.8) is

$$
\mathcal{L}_{0}=-\frac{1}{g_{Y M}^{2}} \sqrt{-g}\left(f(\phi)^{-1} \sqrt{1+f(\phi) g^{\mu \nu} \partial_{\mu} \phi \partial_{\nu} \phi}+V(\phi) \mp f(\phi)^{-1}\right)
$$

where the \pm refers to the $D 3$-brane and $\overline{D 3}$-brane respectively while $f(\phi)=\lambda / \phi^{4}$ as before.

In the presence of four dimensional gravity, there are in general further corrections to the action (4.19) coming from the following considerations. Dynamical $4 d$ gravity leads generically to nontrivial four dimensional curvature $\mathcal{R}$. In effective field theory, this leads to series of contributions of the form

$$
\mathcal{R} \phi^{2}\left(1+c_{1} \mathcal{R} / \phi^{2}+\ldots\right)
$$

More generally each contribution in the action (4.19) could be corrected by terms suppressed by powers of $\mathcal{R} / \phi^{2}$. In the weakly coupled $\mathcal{N}=4 \mathrm{SYM}$ theory coupled to four dimensional gravity, such corrections are evident from diagrams containing loops of W bosons. At strong coupling, such corrections were found in [28, 29] starting from the DBI action for curved slices. From all these points of view, the coefficients $c_{i}$ in 
(4.20) are expected to be of order one (i.e. not parametrically large or small as a function of $\lambda$ ).

In our solutions, we will find that these curvature corrections are negligible. Our strategy will be to simply ignore them at this stage, analyze the dynamics, and then check that this is self consistent (in Section 5.3 below). In any case, we can collect these effects in an effective Lagrangian containing both gravity and matter:

$$
S=\int\left(\frac{1}{2} \sqrt{-g}\left(M_{p}^{2}+\phi^{2}\right) \mathcal{R}+\mathcal{L}_{0}+\ldots\right)
$$

where the ... refers to corrections to the terms in (4.21) which are down by powers of $\mathcal{R} / \phi^{2}$ from the leading terms. The final action is a special case of those considered in $\mathrm{k}$-inflation and k-essence scenarios [3, 4]. In our case, the higher derivative terms in $\mathcal{L}_{0}$ follow from the strong coupling dynamics of the field theory near the origin of its moduli space.

Let us now discuss the reliability and plausibility of this action including the coupling to four dimensional gravity. In string theory, such a coupling is obtained by embedding the AdS geometry into a compactification as a throat emanating from a Calabi Yau compactification of type IIB string theory [26, 27]. The first question that arises is whether this geometrical combination exists or if instead the coupling of the AdS to the Calabi Yau produces a large deformation of the AdS space. If such a geometry exists, we may move onto the second question: whether the low energy effective field theory action is given by (4.19) to a good approximation for the system we wish to study, and what is the order of magnitude of the parameters in the potential $V$. Thirdly, in the end we must check that the time dependent solutions we find are stable against small fluctuations, particle production, and density perturbations. (This is in addition to the checks for small acceleration and small back reaction of the probe energy of the sort we completed in Section 3 for the global case.) This last question will be addressed in section 6 .

As far as the first question goes, as discussed in [26, 27], the AdS throat is obtained from a collection of many D3-branes at a smooth point on the Calabi Yau. At the level of the no scale potential obtained for CY compactifications of type IIB at large radius in the absence of $\alpha^{\prime}$ corrections and nonperturbative effects [27, the scalars on the D3-branes have a potential identical to that in the global quantum field theory on their worldvolume. They are mutually BPS and, in this sense, are also mutually BPS with the rest of the compactification. This follows from the analysis in [27], and may 
be related to the fact that in the $\mathcal{N}=4 \mathrm{SYM}$ at strong coupling, the operator $\operatorname{tr} \Phi^{2}$, which is relevant at weak coupling, is highly irrelevant at strong coupling (something exploited by e.g. [32]). The quantum mechanical corrections to the Kahler potential and the superpotential of the model, which are important for fixing the Kahler moduli, will in general also determine the positions of the D3-branes. Since the configuration corresponding to their being on top of each other at a smooth point in the Calabi Yau is an enhanced symmetry point, it is likely that an order one fraction of the models have a minimum containing to a good approximation an AdS throat arising from such a collection of D3-branes. We will momentarily estimate the size of corrections to this geometry as seen by our probe.

Now let us discuss the second question about the reliability of our effective action and the parameters in the potential. Attached to the Calabi Yau, our AdS throat is coupled to four dimensional gravity and other sectors. We will start by discussing the effects of the coupling of these sectors to $\phi$ in effective field theory. Then we will discuss the geometrical description of these effects. We will argue that (consistently in both descriptions) significant corrections to the mass term in the potential can naturally

arise from these couplings while corrections to the kinetic terms arising from them are subleading to those in the original action. This ensures that we can preserve our slow roll effect for the scalar field coming from the kinetic terms while introducing potential energy sources for gravity which lead to interesting, and in one case accelerating, cosmologies. As we will also discuss, it is an interesting open question whether this setup requires tuning couplings of throat Kaluza Klein modes to other sectors to avoid further effects on the kinetic terms for $\phi$.

\subsubsection{Effective Field Theory Description of Corrections}

As well as the conformal coupling $\mathcal{R} \phi^{2}$ arising from the introduction of gravity, we will generically have further couplings of $\phi$ to other sectors. These could be of the form,

$$
\phi^{2} \eta^{2} \quad \text { or } \quad \frac{\phi^{2}(\partial \eta)^{2}}{M_{*}^{2}}
$$

where $\eta$ is a field from another sector and $M_{*}$ is a mass scale (such as the GUT, string, or Planck scale) in the system above the energies we wish to consider. In the next subsection we will explain why we expect such couplings to be available naturally in string compactifications, but we have not constructed an explicit example and will largely treat these couplings by effective field theory in our analysis of the cosmology. 
We should emphasize that the effective field theory description we use in this subsection makes use of the weak coupling expansion available on the gravity side of AdS/CFT; it is just the couplings of the $\phi$ fields to the new sectors involved in the compactification which we will treat here via effective field theory. A full treatment of the $4 \mathrm{~d}$ effective field theory on the gravity side also requires understanding the couplings and effects of the Kaluza Klein modes. This involves understanding what tuning is required to preserve an (approximate) AdS throat in a Calabi Yau compactification in the absence of a probe brane. This is not well understood. In any case, as we will discuss in the next subsection and in Section 5, the probe approximation for the brane in a large-radius approximate AdS throat is consistent with the presence of a large enough mass on the probe brane for interesting cosmological effects such as inflation.

More generally, we may view (4.22) as coupling the strongly coupled CFT to the $\eta$ sector by hand in effective field theory. The action (4.19) contains no closed string (gravity) loops, or loops of $\eta$ from couplings such as (4.22). We must estimate contributions to our effective actions from these loop effects. We will assume a weak enough string coupling for perturbative string theory to be valid on the gravity side.

Out first important task is to determine the scale of any relevant or marginal corrections to the action that may arise from the couplings involving $\phi$. Let us start by discussing the potential $V(\phi)$, considering a power series expansion

$$
V \equiv V_{0}+V_{2} \phi^{2}+V_{4} \phi^{4}+\ldots
$$

The most relevant term in the Lagrangian at low energies is the hard cosmological constant $V_{0}$. This gets contributions in principle from all sectors in the system. In effective field theory (and approximately in string theory using the Bousso Polchinski mechanism [30]) we may tune this (close) to the value of interest for a given application. We will make use of this freedom in our analysis.

Now let us move on to the $\phi$-dependent couplings, starting with the mass term $V_{2} \phi^{2}$. As we discussed in the global case, in an exact conformal field theory (which is either not coupled to gravity and other sectors or is not destabilized by their presence), a mass coupling $m_{*}^{2} \phi^{2}$ with $m_{*}$ a constant mass parameter is ruled out by the conformal invariance encoded in the effects of the warping in the AdS throat. Thus, with an exact $A d S$ throat geometry, the potential is expected to receive corrections starting with the quartic $V_{4} \phi^{4}$ coupling. However, in general the couplings to gravity and other sectors can generate corrections to the theory (4.21) which violate conformal invariance. For 
example, corrections to the scalar mass coming from loops containing virtual gravitons yield a contribution,

$$
m_{*}^{2} \sim \Lambda_{U V}^{4} / M_{p}^{2}
$$

where the fourth power of the UV loop momentum cutoff $\Lambda_{U V}$ can be traced to the fact that the Feynman diagrams contain derivative couplings. Similar contributions arise from couplings to other sectors (4.22). A mass for $\phi$ can be generated either from a VEV for $\eta$, or from loop corrections involving the derivative coupling, giving rise to respective contributions of the form

$$
m_{*}^{2} \sim\langle\eta\rangle^{2} \quad \text { or } \quad m_{*}^{2} \sim \Lambda_{U V}^{4} / M_{*}^{2}
$$

We should also note that the quartic and higher couplings $V_{4} \phi^{4}+\ldots$ in the effective potential will also get corrections from interactions such as (4.22). In our strategy for obtaining inflation below, we will require the net quartic and higher couplings to be small enough relative to the quadratic coupling. This will not require significant tuning of parameters in our solutions.

Now let us consider the strength of corrections to the crucial generalized kinetic terms in the DBI action (i.e. the series in $v_{p}^{2}=\lambda \dot{\phi}^{2} / \phi^{4}$ ) appearing in the action. Importantly, the corrections to these terms coming from (4.22) are suppressed relative to the existing terms that generate our slow roll effect (in contrast to the situation for the soft mass term just described, for which the (4.25) constitutes the leading effect.) For example, if we compute the effect of the coupling (4.22) on the $\dot{\phi}^{4}$ term in the effective action via a loop of $\eta \mathrm{s}$, we obtain a contribution scaling like

$$
\left(\frac{\dot{\phi}}{M_{*}}\right)^{4} \log \left(\Lambda_{U V} / M_{*}\right)
$$

This is much smaller than the original contribution $N \dot{\phi}^{4} / \phi^{4}$ in the small $\phi$ regime of interest: $\phi^{4} \ll N M_{*}^{4}$.

The fact that the DBI kinetic terms are robust against large corrections from (4.22) arises from the fact that they are marginal and get logarithmic corrections suppressed by the coupling constants in terms like (4.22). There are no soft contributions to the derivative terms in the action-they would have to be of the form $\left(m_{*} / \phi\right)^{n}\left(\dot{\phi}^{2} / \phi^{4}\right)$. Such corrections would be more infrared divergent as $\phi \rightarrow 0$ than the existing ones in (3.8). There are no perturbative diagrams producing such effects, and we find it implausible also in the strong coupling regime that coupling to gravity could worsen the IR behavior of the system. 
Although these corrections to the DBI kinetic terms are small, they are nonzero in general. Since the kinetic terms arose from the DBI action given by the volume of the brane embedded in the ambient geometry, corrections to them correspond to corrections to the AdS geometry at least as seen by our probe brane. Indeed once we generate (or add by hand in effective field theory) a deformation of the theory by a mass term for $\phi$, we expect deviations from exact AdS geometry in the IR. However, as above these deviations are small effects on our probe evolution relative to the effects of the original kinetic terms. In particular, if we deform the Lagrangian to introduce an $m^{2} \phi^{2}$ term, we can estimate its effects on the higher derivative terms in the DBI action. These came from integrating out "W bosons", with the importance of the higher derivative terms arising from the fact that these modes become light at the origin $\phi \rightarrow 0$. The mass term for $\phi$ will at some order induce new contributions to the mass of the $\mathrm{W}$ bosons; up to logarithms these will at most scale like

$$
m_{W}^{2} \rightarrow \phi^{2}+g_{Y M}^{2} m^{2}
$$

In this manner, the contributions of virtual $\mathrm{W}$ bosons to the DBI Lagrangian for $\phi$ become a series in $\lambda \dot{\phi}^{2} /\left(\phi^{2}+g_{Y M}^{2} m^{2}\right)^{2}$. This means that for $\phi \gg g_{Y M}^{2} m$, the evolution is well approximated by that given by the original DBI Lagrangian. As we will see, this constraint preserves a window of interesting behavior for $\phi$ dictated by,

$$
\phi^{2}>g_{s} m^{2}
$$

In Section 5.1, we shall see that the same constraint arises on the gravity side by requiring small back reaction so that the probe approximation holds.

In summary, the same couplings which give rise to a mass $m$ for $\phi$ do not badly alter the generalised kinetic terms for $\phi \gg m$. In fact, the mass term itself is a small effect on the overall probe evolution as we will see from the $\phi$ equation of motion in which the DBI kinetic terms dominate. However, the mass term in the potential will be an important source for the four dimensional spacetime geometry in our cosmological solutions. The corrections on the kinetic terms for $\phi<m$ do mean that ultimately the geometry is probably better approximated by a cut off throat. We will turn to this analysis in subsection 4.2 .

As emphasized above, the four dimensional effective field theory however contains more than just $\phi$ and the BPS W bosons, but also Kaluza Klein modes in the AdS. It is important to check whether couplings similar to (4.22) and the conformal coupling appear with $\phi$ replaced by Kaluza Klein modes. If so, and if the induced masses $m_{K K}$ 
of these Kaluza Klein modes were as big as $m$, the corresponding deformation of the geometry in the IR region would remove our inflationary solution to be described in section 5. In the context of effective field theory, we may tune away these couplings if necessary, and as discussed in the next section we generically expect such tuning to be possible in string theory. It would be interesting to determine whether such tuning is necessary or if $m_{K K} \ll m$ appears naturally.

\subsubsection{Gravity Description of Corrections}

In the previous subsection, we gave arguments based on simple effective field theory couplings that the (soft) mass term for $\phi$ generated by (4.22) will generically be affected more strongly (relative to the original mass term for $\phi$ in the Lagrangian) than the generalized kinetic terms (relative to the original generalized kinetic terms in the Lagrangian). At first sight, this may appear surprising from the point of view of the geometrical picture of a brane probe. Such a probe has kinetic terms determined by the metric of the ambient spacetime, and potential terms introduced via couplings to other background fields (such as the five form RR field strength in the $A d S_{5} \times S^{5}$ solution of type IIB supergravity). There is no general relation between these two effects, though in particular examples they are related in particular ways via the coupled equations of motion for the metric curvature and the other low energy fields. In familiar examples, such as the $A d S_{5} \times S^{5}$ solution, or the Klebanov Strassler solution [31, the strength of the potential is smaller than the effects we found in the previous subsection.

These classical solutions however do not include the effects introduced by the other sectors (mocked up by $\eta$ in the above analysis) located in the bulk of a Calabi Yau to which the throat attaches. In general, the relation between the spacetime metric and the other background fields is different from that obtained in the familiar classical solutions. More general flux backgrounds (including AdS solutions) with different types of brane probes will have a different balance between fluxes felt by the probe and metric curvature due to the contribution of other ingredients. Our results in the above subsection reflect an aspect of this in the context of a coupling of $\phi$ in the familiar solutions to other sectors via, for example, an embedding in a Calabi Yau.

To make this more concrete, let us use known scales of couplings between different sectors of a Calabi Yau geometry to estimate the effective field theory parameters $M_{*}$ and $\Lambda_{U V}$ in the effective field theory analysis of the last subsection. In 33 couplings between brane throats were computed. The results were consistent with couplings between a brane throat and another sector (which could be a much smaller throat) 
suppressed by powers of $M_{*} \sim 1 / R$. Taking the UV cutoff $\Lambda_{U V}$ of the second sector to also be of order $1 / R$, one obtains an estimate for the mass $m$ of order $m \sim 1 / l_{s} \lambda^{1 / 4}$. As discussed in the previous section, the same coupling yields a suppressed correction to the kinetic terms if we lie within the window

$$
\frac{1}{l_{s} \lambda^{1 / 4}}<\phi<\frac{N^{1 / 4}}{l_{s} \lambda^{1 / 4}}
$$

To summarise: the important corrections to the mass term for $\phi$ we derived via effective field theory couplings in the last subsection fit with known properties of geometrical embedding of brane probes, taking into account effects generated by sectors outside the brane throat corresponding to our original CFT. Therefore we find it likely that such a mass term is available (and potentially generic given the presence of a large brane throat) in explicit string models. In any case, a mass for $\phi$ is physically consistent with a probe approximation for $\phi$. This follows from the above considerations regarding the many independent ways of assembling ingredients such as the target space metric and other background fields, combined with the self consistency against back reaction (to be checked in section 5) of the brane energy carried in the $\phi$ mass term.

However, we have not constructed an explicit example of this in a full compactification model. It is a subtle problem to determine the couplings among sectors in a full string compactification [33] 2], and we plan to pursue it systematically in future work.

\subsection{QFT with Mass Gap Coupled to Gravity}

Motivated by the discussion above, we here consider geometries dual to theories with a mass gap, where the AdS throat is cut off at the IR end. The prototypical example of such a geometry is the Klebanov-Strassler solution of 31]. The effects of such an IR cut-off include the following. The warp factor $f(\phi)$ in (3.9) is changed, and further corrections to the action may arise if we start from a cutoff throat at the classical level. Here we shall consider only a toy model in which we focus on the effect on $f(\phi)$; similar comments to those in the previous subsection apply to the question of further corrections to the action.

For the purposes of our discussion, we replace the full Klebanov-Strassler solution with a simple toy model which reproduces the relevant features. We motivate this by considering a theory in which the formula for the $\mathrm{W}$ boson mass (2.2) is deformed to

$$
m_{W}^{2}=\phi^{2}+\mu^{2}
$$


where, in the effective field theory discussion above, we had $\mu \sim g_{Y M}^{2} m$. In general $\mu$ will be related to $m$ in a similar fashion, but for the purposes of this section we keep it arbitrary. To model the W mass (4.29) from the holographic perspective, we keep the action (4.19), but replace the warp factor $f$ with the appropriate function. For simplicity we shall consider,

$$
f(\phi)=\frac{\lambda}{\left(\phi^{2}+\mu^{2}\right)^{2}}
$$

Such a geometry does not satisfy Einstein's equations by itself, but nonetheless exhibits the important features of a capped off throat.

In the DBI Lagrangian (4.19) for the cutoff throat (4.30) in the antibrane case, there is a hard mass of order

$$
m_{D B I}^{2} \sim \mu^{2} / \lambda
$$

Loop corrections arising from the $\phi^{4}$ interactions drive this up to $m_{D B I}^{2} \sim \Lambda_{\phi}^{2} / \lambda$ where $\Lambda_{\phi}$ is the ultra-violet cut-off pertaining to the $\phi$ loop.

In our cutoff geometry we also have corrections to the masses and other couplings as discussed in the last subsection (where now there may be some new sectors associated with the physics at scale $\mu$ ). Again the effects on the kinetic terms are subleading but the mass corrections are important.

\section{Cosmological Solutions}

In Section 3, we saw that the scalar field motion in our system is radically different from the naive expectation based on the finite distance to the origin of the moduli space in the metric appearing in the two derivative action (2.1). Here we shall ask what effects this novel matter sector has on cosmological solutions. We will investigate this in detail by studying the possible FRW cosmologies solutions which follow from (4.19). We consider only spatially flat cosmologies,

$$
d s^{2}=-d t^{2}+a(t)^{2} d x^{2}
$$

Since spatially inhomogeneous terms are redshifted away during inflation, we consider the scalar field ansatz $\phi=\phi(t)$. With this ansatz, the equations of motion can be concisely expressed by first defining the analog of the Lorentz contraction factor in 
special relativity,

$$
\gamma \equiv \frac{1}{\sqrt{1-f(\phi) \dot{\phi}^{2}}}
$$

Our stategy is to analyze the solutions following from (4.19) by first ignoring the effects of the conformal coupling $\mathcal{R} \phi^{2}$, and later showing that its effects are self consistently negligible on our solutions (unlike the situation in usual inflationary models based on (2.1)). The energy density $\rho$ and pressure $p$ following from (4.19) (taking the + sign, corresponding to a D3-brane, for definiteness) are given by,

$$
\begin{aligned}
& \rho=\frac{\gamma}{f}+\left(V-f^{-1}\right) \\
& p=-\frac{1}{f \gamma}-\left(V-f^{-1}\right)
\end{aligned}
$$

These definitions do not include the overall coefficient of $1 / g_{Y M}^{2}$ in (4.19) which instead combines with $M_{p}$ in the Einstein equations so that the scale $\left(M_{p} \sqrt{g_{s}}\right)=M_{p} g_{Y M}$ appears in all equations. The Friedmann equations read,

$$
\begin{aligned}
3 H^{2} & =\frac{1}{g_{s} M_{p}^{2}} \rho \\
2 \frac{\ddot{a}}{a}+H^{2} & =-\frac{1}{g_{s} M_{p}^{2}} p
\end{aligned}
$$

where $H=\dot{a} / a$ is the usual Hubble parameter. Finally, the equation of motion for $\phi$ reads,

$$
\ddot{\phi}+\frac{3 f^{\prime}}{2 f} \dot{\phi}^{2}-\frac{f^{\prime}}{f^{2}}+\frac{3 H}{\gamma^{2}} \dot{\phi}+\left(V^{\prime}+\frac{f^{\prime}}{f^{2}}\right) \frac{1}{\gamma^{3}}=0
$$

where prime denotes a derivative with respect to $\phi$. Note that at as we approach the speed limit on moduli space, so $\gamma \rightarrow \infty$, both the friction term and the potential term in the equation of motion for $\phi$ become subdominant. It is a simple matter to check that the second Friedmann equation (5.36) follows from the first (5.35) together with the equation of motion (5.37). The effective equation of state of our system, defined by $p=\omega \rho$, is therefore given by

$$
\omega=\frac{-\gamma^{-2}-(V f-1) \gamma^{-1}}{1+(V f-1) \gamma^{-1}}
$$




\subsubsection{The Hamilton-Jacobi Formalism}

As occurred in the global case, we will find solutions in which the brane asymptotes to the speed of light at late times in the gravity side background. This means that the quantity $\gamma$ grows as $t$ gets large, and the behavior of $\rho$ will be substantially different from the usual case of $\rho=\frac{1}{2} \dot{\phi}^{2}+V$ to which it reduces for small proper velocity.

Firstly, we want to re-write the Friedmann equations (5.35) and (5.37) in a more tractable form. In fact, they can be integrated once. In the inflation literature this is referred to as the "Hamilton-Jacobi" formalism [34]. Another perspective can be obtained by viewing the resulting cosmology as a Wick rotation of a BPS domain wall; the first order Friedmann equations are related to the Bogomoln'yi equations derived in 35 .

To derive the Hamilton-Jacobi equations the important step is to view the scalar field $\phi$ as the time variable. In practice, this means we consider $H=H(\phi)$ with $\phi=\phi(t)$. This immediately puts a limitation on the dynamics since it assumes $\phi$ is monotonic and $H$ is a single valued function of $\phi$ and therefore does not allow for oscillatory behaviour in $\phi$. We start by taking the time derivative of (5.35) and, using (5.37) along the way, we find

$$
6 H H^{\prime} \dot{\phi}=-\frac{1}{g_{s} M_{p}^{2}} 3 H \gamma \dot{\phi}^{2}
$$

which can clearly be solved simply by

$$
\dot{\phi}=-2\left(g_{s} M_{p}^{2}\right) \frac{H^{\prime}}{\gamma}
$$

Since $\left(\gamma^{-1}\right)$ depends on $\dot{\phi}$, it's useful to invert this to give us

$$
\dot{\phi}=\frac{-2 H^{\prime}}{\sqrt{1 /\left(g_{s}^{2} M_{p}^{4}\right)+4 f H^{\prime 2}}}
$$

Finally, we can substitute this expression for $\dot{\phi}$ into (5.35), using the full expression for $\rho$ from (5.33) to get an expression for the potential $V(\phi)$ in terms of the Hubble parameter $H(\phi)$,

$$
V=3\left(g_{s} M_{p}^{2}\right) H^{2}-\frac{\left(g_{s} M_{p}^{2}\right)}{f} \sqrt{1 /\left(g_{s}^{2} M_{p}^{4}\right)+4 f H^{\prime 2}}+\frac{1}{f}
$$

The advantage of these equations is that they can be solved sequentially. Given a potential $V$, we solve (5.40) to find $H(\phi)$. This can then be plugged into (5.39) to 
find $\phi(t)$. Finally, this can be substituted back into $H(\phi)$ to find the dynamics of the universe. We shall now solve for the late time behaviour of these equations, both in the AdS throat where $f=\lambda / \phi^{4}$, and in our toy model for the cut-off geometry with $f=\lambda /\left(\phi^{2}+\mu^{2}\right)^{2}$.

\subsection{Cosmology in the AdS Throat}

We start by considering cosmology in the CFT, corresponding to a brane moving in the full AdS throat. In this case, the first order Friedmann equations (5.39) and (5.40) read

$$
\dot{\phi}=\frac{-2 H^{\prime}}{\sqrt{1 /\left(g_{s}^{2} M_{p}^{4}\right)+4 \lambda H^{\prime 2} / \phi^{4}}}
$$

and

$$
\lambda V=3 \lambda\left(g_{s} M_{p}^{2}\right) H^{2}-\left(g_{s} M_{p}^{2}\right) \phi^{4} \sqrt{1 /\left(g_{s}^{2} M_{p}^{4}\right)+4 \lambda H^{\prime 2} / \phi^{4}}+\phi^{4}
$$

We will consider potentials of the form

$$
V=V_{0}+V_{2} \phi^{2}+V_{4} \phi^{4}+\ldots
$$

and determine the behaviour of the scale factor as $\phi \rightarrow 0$. For now, we will simply introduce these parameters by hand to analyze the equations but, as mentioned above, there are constraints on these coefficients in the $\mathcal{N}=4$ SYM and in this theory coupled to gravity which we should include in the final analysis. For now however, we work with the ad hoc potential above. There there are three possible cases, depending on which of $V_{0}, V_{2}$ or $V_{4}$ is the first non-vanishing coefficient. (We shall in fact see that if the first two vanish, and the potential is positive, then the result is universal).

\section{Case A: $V_{0} \neq 0$}

If $V$ is simply a constant then equation (5.42) is solved by $H=\sqrt{V_{0} / 3\left(g_{s} M_{p}^{2}\right)}$, in which case equation (5.41) shows that $\phi=$ const, and we get a standard de Sitter phase. Of course, our kinetic terms aren't playing a role here since $\phi$ isn't moving. Note that equation (5.41) requires $V_{0}>0$ here.

Suppose instead that we take $V=V_{0}+V_{4} \phi^{4}$ which suffices to get $\phi$ moving. Then for small $\phi$, we may choose the simple ansatz $H=h_{0}+h_{4} \phi^{4}+\ldots$ where, from (5.42) we 
must choose the coefficients $h_{0}=\sqrt{V_{0} / 3\left(g_{s} M_{p}^{2}\right)}$ and $h_{4}=V_{4} / 6\left(g_{s} M_{p}^{2}\right) h_{0}$. Substituting this into the first of the Friedmann equations, we have the late time behaviour for $\phi$,

$$
\phi \rightarrow \frac{1}{4\left(M_{p} \sqrt{g_{s}}\right) \sqrt{h_{4}}} \frac{1}{\sqrt{t}}
$$

We see that, as in the case of the global CFT considered in Section 3, the scalar field takes infinite time to reach the origin. However, in this case the friction from the expanding universe means that $\phi$ does not saturate the speed limit (2.7). From the late time behaviour of $\phi$, we may immediately determine the late time behaviour of the scale factor. We have,

$$
H=\sqrt{\frac{V_{0}}{3\left(g_{s} M_{p}^{2}\right)}}\left(1+\frac{3}{2^{7} V_{4}\left(g_{s} M_{p}^{2}\right)} \frac{1}{t^{2}}\right)
$$

This result highlights the peculiar cosmologies that can arise due to strong coupling effects of the gauge theory. Here we find a standard de Sitter phase, superposed with a cosmology in which the scale factor has the form $a(t) \sim \exp \left(-c / t M_{p}\right)$. We shall see more cosmologies with this characteristic exponential behaviour in Case D.

Case B: $V_{0}=0$

Let us now consider the interesting case that will give rise to inflation. We suppose that $V_{2} \neq 0$ so that this quadratic mass term dominates at late times when $\phi$ is small.

We work with the simple ansatz,

$$
H=h_{1} \phi+\ldots
$$

Substituting this into (5.42), we get contributions to $V_{2}$ from both the $H^{2}$ term as well the $\sqrt{\cdots}$ term. We find the potential takes the form,

$$
V=\left(3 h_{1}^{2}-\frac{2 h_{1}}{\sqrt{\lambda}}\right)\left(g_{s} M_{p}^{2}\right) \phi^{2}+\mathcal{O}\left(\phi^{4}\right) \equiv V_{2} \phi^{2}+\mathcal{O}\left(\phi^{4}\right)
$$

which, solving for $h_{1}$ in terms of $V_{2}$ gives

$$
h_{1}=\frac{1}{3 \sqrt{\lambda}}\left(1+\sqrt{1+3 V_{2} \lambda /\left(g_{s} M_{p}^{2}\right)}\right)
$$

Substituting our ansatz for $H$ into the equation (5.41), we find

$$
\dot{\phi}=\frac{-2 h_{1} \phi^{2}}{\sqrt{\phi^{4} /\left(g_{s}^{2} M_{p}^{4}\right)+4 \lambda h_{1}^{2}}}
$$


As $\phi \rightarrow 0$, we simply throw away the $\phi^{4}$ term in the denominator to find the late time behaviour

$$
\phi \rightarrow \frac{\sqrt{\lambda}}{t}+\ldots
$$

This coincides with the result (3.13) from the global analysis of Section 3; in this theory the slowing down of the scalar field is driven by the speed limit rather than friction from the expanding spacetime. We now substitute this back into our ansatz (5.44) to find the late time behaviour of the scale factor,

$$
a(t) \rightarrow a_{0} t^{h_{1} \sqrt{\lambda}}
$$

Using (5.46), the exponent is

$$
h_{1} \sqrt{\lambda}=\frac{1}{3}\left(1+\sqrt{1+\frac{3 V_{2} \lambda}{\left(g_{s} M_{p}^{2}\right)}}\right) \approx \sqrt{\frac{V_{2} \lambda}{3\left(g_{s} M_{p}^{2}\right)}}
$$

where the approximation is true only for $V_{2} \lambda \gg g_{s} M_{p}^{2}$. We see that we can orchestrate an inflationary universe if the potential $V_{2}$ is sufficiently large. Specifically

$$
\frac{V_{2} \lambda}{g_{s} M_{p}^{2}}>1
$$

It is amusing to recall that usual slow roll inflation occurs only if the potential is suitably flat. Here we find the opposite result: with a speed limit on the scalar field, inflation occurs only if $V_{2}$ corresponds to a high enough mass scale (we see from (5.49) that this mass scale can be much lower than $M_{p}$, but the acceleration gets stronger for larger $\left.V_{2}\right)$.

Interestingly, we have obtained a form of power law inflation, since the scale factor behaves as a power of $t$. However it arises in the case of a polynomial (quadratic) potential, which under the usual circumstances (2.1) leads to exponential inflation $a \sim e^{H t}$. (The potential required to obtain power law inflation from (2.1) is exponential in $\phi$.)

Naively it appears that this result suggests an alternative to the usual quintessence scenario where a scalar field rolls towards an asymptotic regime of moduli space. Here the scalar field could roll a finite distance in the field theory moduli space metric in the interior of the moduli space and still lead to asymptotic acceleration. However, we are limited to a finite period of controlled speed of light evolution because of the 
back reaction of the probe on the geometry. In the global case we determined this backreaction in (3.17). It is a simple matter to repeat the calculation here where the energy of the brane is dominated by the potential $V_{2} \phi^{2}$. Once again, translating from the proper energy $E_{p}$ to the observers energy density $E=E_{p}(r / R)^{4}$, we have the constraint

$$
E_{p} l_{5}^{3} \sim \frac{V_{2} \phi^{2}}{g_{Y M}^{2}}\left(\frac{R}{r}\right)^{4} \frac{\alpha^{\prime 4} g_{s}^{2}}{R^{5}}<\frac{1}{R}
$$

which gives us the constraint that our solution is only to be trusted for $\phi$ greater than

$$
\phi^{2}>g_{s} V_{2}
$$

Notice that this is identical to the lower bound on $\phi$ arising derived in Section 4 by calculating one-loop corrections to the W-boson mass using effective field theory methods (4.27). Despite this infra-red cut-off to our inflationary phase, we shall shortly argue that we still retain a viable window of inflation in this scenario.

Case C: $V_{0}=0$ and $V_{2}=0$

Let us now suppose that the potential has only $V_{4} \neq 0$. As we have seen, such a potential is generated by a $\overline{D 3}$-brane moving in the AdS throat. The late-time analysis of the Hamilton-Jacobi equations (5.41) and (5.42) is actually just a special case of the above discussion, where we put $h_{1}=2 / 3 \sqrt{\lambda}$. We can read of the final result from (5.47): we have

$$
a \rightarrow a_{0} t^{2 / 3}
$$

which looks like dust. In fact, for any potential for which $V_{0}=V_{2}=0$ with higher order terms non-vanishing, the speed limit on the moduli space ensures that the rolling scalar field looks has equation of state $\omega \approx 0$ : a kinetic dust.

Case D: $V<0$

To find equations of state with $\omega>0$, we require negative $V_{2}$. From (5.46), we see that for $0<h_{1}<2 / 3 \sqrt{\lambda}$ we have

$$
-\lambda\left(g_{s} M^{2}\right)^{2} \frac{\lambda}{3}<V_{2}<0
$$

The late time behaviour of the scale factor is once again given by (5.47). During this expansion, the scalar field is rolling up the inverted harmonic oscillator potential. Without the higher kinetic terms, the scalar would overshoot the top and roll down the other side. However, the speed limit does not allow this to happen, and slows the field to prevent it reaching the top at $\phi=0$ in finite time. 
Let us now consider the case $V_{2}=0$ with $V_{4}<0$. We can generate such a potential by considering, for example, $H=h_{3} \phi^{3}$ which gives rise to the potential $V=V_{4} \phi^{4}+V_{6} \phi^{6}$ where

$$
\lambda V_{4}=-\sqrt{1+36 \lambda h_{3}^{2}\left(g_{s}^{2} M_{p}^{4}\right)}+1 \quad, \quad \lambda V_{6}=3 \lambda h_{3}^{2}\left(g_{s} M_{p}^{2}\right)
$$

At late times the scalar field rolls like $\phi \sim 1 / t$, and the equation of state parameter diverges: $\omega \rightarrow \infty$. Meanwhile, the scale factor $a$ has the peculiar behaviour

$$
a=a_{0} \exp \left(-\frac{\left(1 /\left(g_{s} M_{p}^{2}\right)+36 \lambda h_{3}^{2}\right)^{3 / 2}}{2^{4} 3^{3} h_{3}^{2}} \frac{1}{t^{2}}\right)
$$

which undergoes a period of accelerated expansion before settling down to a steadystate cosmology at late times. The period of acceleration coincides with the period when the $V_{6} \phi^{6}$ term dominates over the $V_{4} \phi^{4}$ term in the potential; in the solution this also correlates with a period in which the potential dominates in the gravitational dynamics determined by $\omega$ (5.38). As in case B, the effect becomes stronger with a stronger potential (so a flat potential is not required for slow roll since this is provided by the DBI kinetic terms).

Scale factors with the characteristic $\exp \left(-1 / t^{2}\right)$ behaviour also occur in standard cosmology, but only for very finely tuned inital conditions. The novelty here is that such dynamics is generic for a large enough intitial velocity. A similar steady state dynamics also arises from $H=h_{4} \phi^{4}$, this time with $a \sim \exp \left(1 / M_{p} t\right)$. Each of these scenarios finds Xeno exonerated and Hoyle happy.

\subsection{Cosmology in the Cut-Off Throat}

The interesting Case $\mathrm{B}$ of the previous section has $V_{2} \neq 0$ which is not compatible with the conformal symmetry implied by an exact AdS throat. Instead, one ultimately expects a geometry in which the throat is cut off in the infra-red. We described such geometries in Section 4. Here we will work with the toy model in which the warp factor is taken to be

$$
f(\phi)=\frac{\lambda}{\left(\phi^{2}+\mu^{2}\right)^{2}}
$$

For $\phi \gg \mu$, the geometry is essentially that of the AdS throat and the analysis of the previous sections holds. However, for $\phi \leq \mu$, the speed limit becomes simply

$$
\dot{\phi} \leq \frac{\mu^{2}}{\sqrt{\lambda}}
$$


So the scalar field can now reach the origin in finite time and happily sail right through at finite speed. This has the advantage that at late times the field oscillates around the minimum of the potential as usual and reheating may occur (depending on the couplings to the Standard Model). However, we must check that we can still arrange for a period of acceleration. The condition for acceleration requires large enough $V_{2}$ as we saw in (5.49) Generally we have

$$
V_{2}=m^{2}
$$

where masses $m^{2}$ which could be generated in various ways were listed in Section 4 and include contributions from graviton loop (4.24), couplings to other sectors (4.25) and a hard mass from the deformed geometry (4.31).

Let us start by examining the upper limit for $\phi$. In fact, we are quite entitled to take $\phi$ as large as we wish and, for $\phi \gg M_{p}$, one finds the standard slow roll expansion of chaotic inflation. However, as we shall discuss in Section 5.3, models with superPlanckian VEVs suffer from destabilization from a slew of quantum corrections involving for example gravitational conformal couplings $\mathcal{R} \phi^{2}$ [2]. To avoid this, we shall instead restrict ourselves to subPlanckian VEVs

$$
\phi \ll M_{p},
$$

where, as the speed limit is saturated, we may still obtain inflationary behaviour.

In our cut-off geometry we also need $\phi$ to be suitably large so that the probe brane is experiencing the $A d S$ throat-like background before the cut-off is reached. It can be checked that the solution described in Case B of Section 5.1 continues to hold in the cut-off geometry provided $\phi \gg \mu$. We therefore find our window of inflation when the scalar field lives in the regime,

$$
\mu \ll \phi \ll M_{p}
$$

during which time we have the cosmological solution $a(t)=a_{0} t^{h_{1} \sqrt{\lambda}}$ while the scalar field slows down as $\phi=\sqrt{\lambda} / t$. Let us assume that this solution actually holds all the way to the end points (5.51). This may be overly optimistic, but it gives us a quick and ready way to calculate the number of e-foldings. We require that at the initial time $t=t_{0}$ we have $\phi\left(t_{0}=0\right)=M_{p}$, while at the final time $t=t_{f}$ we have $\phi\left(t=t_{f}\right)=\mu$. Then,

$$
t_{0}=\frac{\sqrt{\lambda}}{M_{p}} \quad \text { and } \quad t_{f}=\frac{\sqrt{\lambda}}{\mu}
$$


The number of e-foldings is therefore given by,

$$
n=\log \left(\frac{a\left(t=t_{f}\right)}{a(t=0)}\right)=h_{1} \sqrt{\lambda} \log \left(M_{p} / \mu\right)
$$

The argument of the logarithm is roughly the distance travelled by the scalar field. Any large number of efoldings comes from the prefactor. Using (15.48) we have

$$
n \sim h_{1} \sqrt{\lambda} \sim \frac{m \sqrt{\lambda}}{\sqrt{g_{s}} M_{p}} \geq 100
$$

This criterion is the same as the criterion that the potential $V$ dominate in the equation of state (in particular in $\rho$ ).

This inflationary phase is novel in several ways. Most importantly, we find inflation to occur at sub-Planckian field strength for $\phi$. We will show in section 5.3 that, when combined with the novel form of the equation of motion for $\phi$, this allows us to avoid destabilizing effects from the conformal coupling $\mathcal{R} \phi^{2}$, circumventing some of the difficulties involved in placing inflation within a string compactification 2]. Moreover, as noted in Section 5.1, we have obtained power-law inflation from a quadratic potential.

At later times, the brane reaches the end of the cutoff throat and the novelties of the DBI action wear off. At this stage, we expect to reduce to an ordinary matter or radiation dominated phase, though we have not yet controlled the details of the exit from our inflationary stage.

The number of e-foldings (5.52) can be made large if we can generate a large mass term $m^{2} \phi^{2}$ for our scalar field. In Section 4, we discussed possible mechanisms for generating such a term and argued that corrections to our crucial higher derivative terms were sub-leading. The simplest of the mass corrections was the hard contributions (4.31) from the DBI action itself: $m_{D B I} \sim \mu / \sqrt{\lambda}$. Comparing with (5.52) we immediately see that in our strong coupling regime $\lambda \gg 1$ there is no inflationary window with such a mass: we need another mechanism. As discussed at length in Section 4, such a mechanism may arise from coupling to other sectors which (depending on the strength of the couplings and cutoff scales for the other sectors in the system) may produce a large enough mass. More simply, as discussed above we may consider deforming the theory by a mass term for $\phi$ and note that this induces only subdominant corrections to the DBI kinetic terms. Again, it would be very interesting to determine the magnitude of mass $m$ arising in specific string compactifications. 


\section{A Comment on $V_{4}$ Terms}

In the above discussion, we concentrated on the possible mass terms that could be generated, and the associated window of applicability of the inflationary solution described in Case B of Section 5.1. However, the solution itself is valid only if the quadratic term in the potential dominates over the quartic term; otherwise we obtain the dust-like evolution of Case C. We therefore require,

$$
V_{4} \phi^{2}<m^{2} \Rightarrow \phi^{2}<m^{2} / V_{4}
$$

If $V_{4}$ is too big, then this constraint on $\phi$ would be much stronger than (5.50) and potentially ruin our window of inflation. Let us therefore examine the possible $V_{4}$ couplings.

In the DBI action, $V_{4}$ scales like $1 / \lambda$. However, quantum corrections change this. Let's focus on the virtual effects of other sectors, since these were successful in giving us an inflationary window. The derivative coupling (4.22), leads to a contribution of order

$$
\Delta V_{4} \sim \Lambda_{U V}^{4} / M_{*}^{4}
$$

while at the same time inducing a mass $m_{*} \sim \Lambda_{U V}^{2} / M_{*}$ (4.25) as we discussed earlier. Plugging this into (5.53) leads to the constraint $\phi<M_{*}$ which is stronger than (5.50). At the level of our effective field theory analysis (in terms of an unknown UV cutoff parameter $\Lambda_{U V}$ and a coupling scale $1 / M_{*}$ ), this is consistent with our inflationary window for appropriate values of these parameters.

\subsection{The Conformal Factor and Curvature Corrections}

Let us now restore into our analysis the conformal coupling $\mathcal{R} \phi^{2}$ and the other curvature corrections scaling as powers of $\mathcal{R} / \phi^{2}$. We will consider the regime of our solutions where $\phi \ll M_{p}$, and show that the solutions are not destabilized by the addition of these curvature couplings.

Let us first consider the $\mathcal{R} / \phi^{2}$ corrections to the terms in the Lagrangian (4.21) which scale like $H^{2} / \phi^{2}$. In our inflating solution, these terms are of the order $m^{2} / M_{p}^{2}$. As long as this quantity is sufficiently smaller than 1, then the correction terms are negligible compared to the terms in (4.21) that we did include in the initial analysis. In the noninflating solutions, $H \sim 1 / t$ and these corrections are suppressed by powers of $\lambda$ which makes them very safe from affecting the results above. 
Now let us turn to the conformal coupling $\mathcal{R} \phi^{2}$. First, let us consider the Einstein equations for the metric. The effect of the conformal coupling is to replace $M_{p}^{2}$ with $M_{p}^{2}+\phi^{2}$ in these equations, changing the effective Planck mass. Since $\phi \ll M_{p}$, this does not change the solutions for $a(t)$ significantly. Similar comments apply to the $H^{2} \phi^{2}$ terms which plague F-term inflation in supergravity models.

Secondly, we must check whether the scalar field equation of motion is self consistently solved by the original solution in the presence of a conformal coupling. The coupling $\xi \mathcal{R} \phi^{2}$ contributes to the equation of motion for $\phi$, (5.37), as

$$
\frac{\lambda \ddot{\phi}-6 \lambda \dot{\phi}^{2} / \phi+4 \phi^{3}}{\left(1-\lambda \dot{\phi}^{2} / \phi^{4}\right)^{3 / 2}}+\frac{3 \lambda H \dot{\phi}}{\left(1-\lambda \dot{\phi}^{2} / \phi^{4}\right)^{1 / 2}}+\left(V^{\prime}+2 \xi \mathcal{R} \phi-4 \phi^{3}\right)=0
$$

Because $\gamma(t)^{-1}=\sqrt{1-\lambda \dot{\phi}^{2} / \phi^{4}}$ is going to zero at late times, the terms involving $V$ and $\mathcal{R}$ are subdominant in this regime. This is reflected in the fact that, for Cases $\mathrm{B}$ and $\mathrm{C}$ of Section 5.1, the late time behaviour of the scalar field saturates the speed limit and is independent of the potential.

In summary, the conformal coupling and other curvature couplings do not change our results. This is in contrast to the usual situation (2.1), where for example the slow roll conditions for inflation in a polynomial potential are impossible to satisfy in the presence of the conformal coupling. This point was emphasized for the case of 3 -branes in warped throats in [2. Here see that near the origin, where the DBI velocity corrections are crucial, the structure of the scalar equation of motion is dramatically different due to the factors of $\gamma(t)$ in the denominator in (5.55).

\subsection{Relation to other Works}

The behavior of our system, especially in Case C in Section 5.1, bears a strong resemblance to the tachyon matter system studied in recent years [5, 8]. Indeed, the anti-brane moving in the $A d S$ throat is, in some sense, a strong coupling limit of the tachyon matter system. There are important differences between our case and the weakly coupled tachyon matter system, most notably the fact that as we discussed above in our situation the tachyon has been lifted and, as we shall see, particle production is suppressed. Moreover, the spectra of particles and strings in the two backgrounds differ. 
A simple field redefinition can be used to relate the effective field theories in the two cases as follows. In tachyon cosmology, the action for the tachyon is of the form [36]

$$
\mathcal{L}=-F(T) \sqrt{1-\dot{T}^{2}}
$$

which, upon expanding the square-root, has a potential term $F(T)$. We can simply generalise this action to include a further potential term $G(T)$, which we could use either to cancel $F$ or simply to make the potential different from the coefficient of the kinetic terms. Such a potential may be generated in situations where the brane antibrane system is embedded in a compactification. We therefore consider,

$$
\mathcal{L}=-F(T) \sqrt{1-\dot{T}^{2}}-G(T)
$$

The stress-energy tensor and pressure can be easily calculated to yield energy density

$$
\rho=\frac{F}{\sqrt{1-\dot{T}^{2}}}+G \quad, \quad p=-F \sqrt{1-\dot{T}^{2}}-G
$$

Tachyon cosmologists work with this Lagrangian imposing $G=0$. Of particular relevance for the present work is the observation 9] that the power-law inflationary phase of Case B can be obtained by the choice $F(T) \sim 1 / T^{2}$ in the Lagrangian (5.56). It was further shown that higher order (inverse) potentials give rise to dust behaviour as we saw in Case C. To return to our favourite Lagrangian and compare these results with those above, we employ the field redefinitions

$$
T=\frac{\sqrt{\lambda}}{\phi} \quad, \quad F(T)=\frac{\lambda^{2}}{T^{4}} \quad, \quad G(T)=V(\phi)-\phi^{4}
$$

It is very interesting to ask how generally motion on internal scalar field configuration spaces can be imbued with a geometric interpretation (and therefore a speed limit induced by causality). It is intriguing that the tachyon Lagrangian (5.56) has a structure reminiscent that of a relativistic particle moving in spacetime.

The motion of the probe brane in the cut-off geometry is also somewhat similar to the discussion of [37. These authors consider the induced mirage cosmology [38] on a probe brane as it moves in the Klebanov-Strassler geometry [31]. This differs from our analysis in two important respects: firstly, in [37] the probe brane was taken to move slowly through the background geometry, so that the DBI action could be approximated by two-derivative terms. Secondly, the cosmology was viewed from an observer on the probe brane, rather than from the perspective of a boundary observer as is relevant for our discussion. 


\section{Perturbations and Particle Production}

We now come to the promised self consistency checks of our time dependent evolution. In order to trust our results in the previous sections, we must check that particle and string production and fluctuations about the solution do not destabilize it. We have already checked that acceleration is small enough to avoid closed string production, and that back reaction from the probe's large kinetic energy is small in the window (3.17).

\subsection{W boson and String Production}

Firstly, we consider the production of massive strings on the brane and W-bosons. Our effective DBI action is blind to the possible on-shell creation of these modes and we must check by hand that the production is suppressed in the $\lambda \gg 1$ regime. This is rather simple. The time dependent solution we found entails time dependent masses for both W-bosons (2.2) and massive string modes on the brane (2.6) since these masses are proportional to $\phi \sim \sqrt{\lambda} / t$.

The strength of particle production for a time dependent frequency $\omega(t)$ is controlled by $\dot{\omega} / \omega^{2}$. This is a rough estimate which can be obtained by starting from the requirement for adiabatic evolution for a frequency which changes over a time period $\delta t$ :

$$
\delta t \gg 1 / \omega_{\min }
$$

where $\omega_{\text {min }}$ is the smallest value of $\omega$ obtained in the evolution. Dividing by the change $\delta \omega$ arising in the process yields

$$
\delta t / \delta \omega \gg 1 /\left(\omega_{\min } \delta \omega\right)
$$

Consider a long enough evolution so that $\delta \omega$ is of order $\omega_{\max }$. Replacing $\delta \omega$ on the right hand side by $\omega_{\max }$ and replacing $\omega_{\max } \omega_{\min }$ by $\omega^{2}$ yields a condition under which particle production may be consistently neglected:

$$
\dot{\omega} \ll \omega^{2}
$$

This can be thought of as expressing the fact that (on average) the energy from the time dependence of the frequency is smaller than the jump in energy needed to produce an on shell particle. We find in our situation that the inequality (6.59) is satisfied parametrically in $\lambda$ for large $\lambda$. In particular for the $W$ boson zero modes we find

$$
\frac{\dot{m}_{W}}{m_{W}^{2}} \sim \frac{1}{\sqrt{\lambda}}
$$


which is suppressed in our large $\lambda$ regime. Nonzero modes are more suppressed. We also need to check the production of open string oscillator modes on the brane. The masses for these modes are given by the warped value $m_{s}(\phi) \sim \phi / \lambda^{1 / 4}$. If they were created at rest, a similar calculation to the one above would yield again parametric suppression of $\dot{m}_{s} / m_{s}^{2} \sim 1 / \lambda^{1 / 4}$. Since the brane is moving with a large velocity $\sqrt{\lambda} \dot{\phi} / \phi^{2}$, the strings created on the brane have energies boosted to

$$
\omega \sim \gamma \sqrt{N_{o s c} m_{s}(\phi)^{2}+\vec{p}^{2}}
$$

where $N_{\text {osc }}$ is an integer coming from the oscillator level of the string. These energies are large, and we find that again the time dependent background does not inject enough energy to create these states. Therefore our solution is stable against string and $\mathrm{W}$ boson production in the strong coupling regime.

\subsection{Perturbations of $\phi$}

In the remainder of this section, we will analyze the fluctuations of $\phi$ itself about the solutions we have found. Our main goal will be to determine in what regime the perturbations are not dangerous for our solutions above. These results also pertain to the spectrum of density perturbations produced in our inflationary phase. We will defer a detailed discussion of any predictions of that model for the CMBR to a later investigation. We perturb our solutions as,

$$
\phi(t) \rightarrow \phi(t)+\alpha(x, t)
$$

Expanding in Fourier modes, $\alpha(x, t)=\alpha_{k}(t) e^{i k x}$, the equation of motion for $\alpha$ obtained by expanding the action (4.19) is,

$$
\ddot{\alpha}_{k}+\left(\frac{6}{t}+3 H\right) \dot{\alpha}_{k}+\left(\frac{6}{t^{2}}+\frac{6 H}{t}+\frac{k^{2}}{\gamma_{0}^{2} a^{2} t^{4}}\right) \alpha_{k}=0
$$

where we have used the late time behavior of the function $\gamma$ defined in (5.32) which, for most of our solutions, is $\gamma \rightarrow t^{2} / \gamma_{0}$ for constant $\gamma_{0}$. In particular, for the inflationary solution of Case B we have $\gamma_{0} \sim m M_{p} / \sqrt{\lambda}$.

If we analyze this equation of motion in the global case, where $H=0$, we obtain the results $\delta \phi \propto t^{-2}$ and $\delta \phi \propto t^{-3}$ for the $k=0$ mode. For the $k \neq 0$ modes, the perturbations will be further suppressed. So we see that the perturbations do not grow relative to the background solution $\phi \sim \sqrt{\lambda} / t$; i.e. these perturbations are non-tachyonic. 
Let us now analyze the equation with gravity turned on, focusing on the inflationary solution. We would like to estimate the density perturbations that result from the fluctuation $\delta \phi$ in the field. In our inflationary phase, we have

$$
H=\frac{1}{\epsilon_{0} t}
$$

where, from (5.47), we have $\epsilon_{0}=\left(h_{1} \sqrt{\lambda}\right)^{-1}$. Since we have $\epsilon_{0} \ll 1$, in the inflationary phase the terms involving $H$ and $H / t$ dominate over the terms involving $1 / t$ and $1 / t^{2}$ respectively. The relative importance of the $k$-terms depends on the wavelength under consideration. In general, we therefore have

$$
\ddot{\alpha}_{k}+\frac{3}{\epsilon_{0} t} \dot{\alpha}_{k}+\left(\frac{6}{\epsilon_{0} t^{2}}+\frac{c k^{2} \lambda}{M_{p}^{2} m^{2}} \frac{1}{a^{2} t^{4}}\right) \alpha_{k}=0
$$

where $c$ is a constant of order one, parameterising our ignorance of $\gamma_{0}$.

For superhorizon fluctuations, defined by $k^{2} \ll a^{2} H^{2}$, where $a H$ is the comoving horizon size, we may neglect the spatial kinetic terms in (6.63). This leads to a simple pair of solutions to the classical equation (6.63) for the modes:

$$
\alpha^{(0)} \sim \frac{\alpha_{0}(k)}{t^{2}}
$$

and

$$
\alpha^{(1)} \sim \frac{\alpha_{1}(k)}{t^{3 / \epsilon_{0}}}
$$

These modes decay faster at large $t$ than the perturbations of the scalar field in ordinary slow roll inflation based on (2.1), which go to a constant as the modes cross the horizon. There are reasonable regimes of parameters for which all the subPlanckian $k / a$ modes have the gradient term in (6.63) suppressed relative to the effective mass squared term there.

To obtain a prediction for the observed density perturbations, we must be careful to follow them as they re-enter the horizon at later times. For now, let us simply check that the perturbations do not destabilize our inflationary phase.

During inflation, the density perturbations are simply

$$
\left.\frac{\delta \rho}{\rho}\right|_{k, \text { during inflation }} \sim \frac{V^{\prime} \delta \phi_{k}}{V}
$$


For our polynomial potentials, this yields $\delta \phi / \phi$. As we have seen, our perturbations die faster than in ordinary inflation due to the stronger role for the mass term in (6.63), and they certainly do not compete with $\phi$ in magnitude. Thus our density perturbations during inflation are small enough to avoid back reaction. The question of whether one obtains eternal inflation depends on the magnitude of $H \delta \phi / \dot{\phi}$, which can be small in our speed of light phase. We plan to pursue in future work a detailed study of the predictions of our inflationary models, as well as an analysis of reheating and other required features.

\section{Acknowledgements}

At the risk of sounding like overly-grateful oscar recipients, we would like to thank B. Acharya, T. Banks, S. Cherkis, N. Constable, E. Copeland, M. Fabinger, D. Freedman, A. Guth, M. Headrick, S. Kachru, B. Kors, N. Lambert, A. Linde, H. Liu, J. Maldacena, E. Martinec, J. Minahan, C. Nunez, H. Ooguri, M. Peskin, J. Polchinski, M. Schnabl, S. Shenker, W. Taylor, J. Troost and D. Wands for useful and interesting discussions. And, of course, everyone else who knows us. We are grateful to the organizers and participants of the 2003 Benasque string theory workshop for providing a stimulating environment where this project was initiated. Thanks also to the organizers and participants of the KITP string cosmology workshop for hospitality and useful advice on some of the issues in this paper, and to those of the 2003 International String School and Workshop in Iran, where these results were first unveiled. D.T. would like to thank SLAC for hospitality while this work was completed. E.S. is supported in part by the DOE under contract DE-AC03-76SF00515 and by the NSF under contract 9870115. This research was supported in part by the National Science Foundation under Grant No. PHY99-07949. D. T. is supported by a Pappalardo fellowship, and is grateful to Neil Pappalardo for his generosity. This work was also supported in part by funds provided by the U.S. Department of Energy (D.O.E.) under cooperative research agreement \#DF-FC02-94ER40818.

\section{References}

[1] D. Kabat and G. Lifschytz, "Gauge theory origins of supergravity causal structure" JHEP 9905, 005 (1999) [arXiv hep-th/9902073].

[2] S. Kachru, R. Kallosh, A. Linde, J. Maldacena, L. McAllister and S. P. Trivedi, "Towards inflation in string theory" arXiv hep-th/0308055.

[3] C. Armendariz-Picon, T. Damour and V. Mukhanov, "k-inflation" Phys. Lett. B 458, 209 (1999) [arXiv hep-th/9904075. 
[4] T. Chiba, T. Okabe and M. Yamaguchi, "Kinetically driven quintessence" Phys. Rev. D 62, 023511 (2000) [arXiv astro-ph/9912463].

C. Armendariz-Picon, V. Mukhanov and P. J. Steinhardt, "A dynamical solution to the problem of a small cosmological constant and late-time cosmic acceleration", Phys. Rev. Lett. 85, 4438 (2000) [arXiv astro-ph/0004134].

C. Armendariz-Picon, V. Mukhanov and P. J. Steinhardt, "Essentials of k-essence" Phys. Rev. D 63, 103510 (2001) [arXiv astro-ph/0006373.

[5] A. Sen, "Rolling tachyon" JHEP 0204, 048 (2002) [arXiv hep-th/0203211.

A. Sen, "Tachyon matter" JHEP 0207, 065 (2002) [arXiv hep-th/0203265].

[6] A. Maloney, A. Strominger and X. Yin, "S-brane thermodynamics" arXiv:hep-th /0302146.

[7] N. Lambert, H. Liu and J. Maldacena, "Closed strings from decaying D-branes", arXiv hep-th/0303139.

[8] G. W. Gibbons, "Cosmological evolution of the rolling tachyon" Phys. Lett. B 537, 1 (2002) [arXiv hep-th/0204008.

[9] A. Feinstein, "Power-law inflation from the rolling tachyon", Phys. Rev. D 66, 063511 (2002) [arXiv hep-th/0204140.

T. Padmanabhan, "Accelerated expansion of the universe driven by tachyonic matter" Phys. Rev. D 66, 021301 (2002) [arXiv hep-th/0204150.

L. R. Abramo and F. Finelli, "Cosmological dynamics of the tachyon with an inverse power-law potential", arXiv astro-ph/0307208.

[10] J. H. Horne and G. W. Moore, "Chaotic coupling constants" Nucl. Phys. B 432, 109 (1994) [arXiv hep-th/9403058.

[11] T. Banks, M. Berkooz, S. H. Shenker, G. W. Moore and P. J. Steinhardt, "Modular cosmology" Phys. Rev. D 52, 3548 (1995) [arXiv:hep-th/9503114.

[12] T. Banks, W. Fischler and L. Motl, "Dualities versus singularities", JHEP 9901, 019 (1999) [arXiv hep-th/9811194.

[13] F. Quevedo, "Lectures on string / brane cosmology" Class. Quant. Grav. 19, 5721 (2002) [arXiv; hep-th/0210292.

[14] C. Bachas, "D-brane dynamics", Phys. Lett. B 374, 37 (1996) [arXiv hep-th/9511043.

[15] M. R. Douglas, D. Kabat, P. Pouliot and S. H. Shenker, "D-branes and short distances in string theory", Nucl. Phys. B 485, 85 (1997) [arXiv hep-th/9608024.

[16] C. P. Burgess, P. Martineau, F. Quevedo and R. Rabadan, "Branonium", JHEP 0306, 037 (2003) [arXiv hep-th/0303170.

[17] M. Brandle and A. Lukas, "Flop transitions in M-theory cosmology", Phys. Rev. D 68, 024030 (2003) [arXiv:hep-th/0212263.

[18] L. Jarv, T. Mohaupt and F. Saueressig, "M-theory cosmologies from singular Calabi-Yau compactifications", arXiv:hep-th/0310174.

[19] I. L. Buchbinder, A. Y. Petrov and A. A. Tseytlin, "Two-loop $N=4$ super Yang Mills effective action and interaction between D3-branes" Nucl. Phys. B 621, 179 (2002) [arXiv:hep-th/0110173. 
[20] O. Aharony, S. S. Gubser, J. M. Maldacena, H. Ooguri and Y. Oz, "Large N field theories, string theory and gravity" Phys. Rept. 323, 183 (2000) [arXiv:hep-th/9905111.

[21] L. Randall and R. Sundrum, "A large mass hierarchy from a small extra dimension" Phys. Rev. Lett. 83, 3370 (1999) [arXiv hep-ph/9905221.

[22] J. M. Maldacena, "The large $N$ limit of superconformal field theories and supergravity" Adv. Theor. Math. Phys. 2, 231 (1998) [Int. J. Theor. Phys. 38, 1113 (1999)] [arXiv:hep-th/9711200].

[23] P. C. Aichelburg and R. U. Sexl, "On The Gravitational Field Of A Massless Particle" Gen. Rel. Grav. 2, 303 (1971).

[24] A. Sen, "Open-closed duality: Lessons from matrix model" arXiv hep-th/0308068.

[25] S. Kachru, J. Pearson and H. Verlinde, "Brane/flux annihilation and the string dual of a non-supersymmetric field theory", JHEP 0206, 021 (2002) [arXiv hep-th/0112197].

[26] H. Verlinde, "Holography and compactification" Nucl. Phys. B 580, 264 (2000) [arXiv: hep-th/9906182.

[27] S. B. Giddings, S. Kachru and J. Polchinski, "Hierarchies from fluxes in string compactifications" Phys. Rev. D 66, 106006 (2002) [arXiv hep-th/0105097.

[28] N. Seiberg and E. Witten, "The D1/D5 system and singular CFT" JHEP 9904, 017 (1999) [arXiv hep-th/9903224.

[29] A. Fotopoulos and A. A. Tseytlin, "On gravitational couplings in D-brane action" JHEP 0212, 001 (2002) [arXiv hep-th/0211101].

[30] R. Bousso and J. Polchinski, "Quantization of four-form fluxes and dynamical neutralization of the cosmological constant", JHEP 0006, 006 (2000) [arXiv hep-th/0004134.

[31] I. R. Klebanov and M. J. Strassler, "Supergravity and a confining gauge theory: Duality cascades and $\chi S B$-resolution of naked singularities" JHEP 0008, 052 (2000) [arXiv:hep-th/0007191].

[32] M. J. Strassler, "Non-supersymmetric theories with light scalar fields and large hierarchies" arXiv hep-th/0309122.

[33] S. Dimopoulos, S. Kachru, N. Kaloper, A. E. Lawrence and E. Silverstein, "Small numbers from tunneling between brane throats" Phys. Rev. D 64, 121702 (2001) [arXiv:hep-th/0104239.

S. Dimopoulos, S. Kachru, N. Kaloper, A. E. Lawrence and E. Silverstein, "Generating small numbers by tunneling in multi-throat compactifications", arXiv hep-th/0106128.

[34] A. G. Muslimov, "On The Scalar Field Dynamics In A Spatially Flat Friedman Universe" Class. Quant. Grav. 7, 231 (1990).

D. S. Salopek and J. R. Bond, "Nonlinear Evolution Of Long Wavelength Metric Fluctuations In Inflationary Models", Phys. Rev. D 42, 3936 (1990).

W. H. Kinney, "A Hamilton-Jacobi approach to non-slow-roll inflation" Phys. Rev. D 56, 2002 (1997) [arXiv hep-ph/9702427.

[35] O. DeWolfe, D. Z. Freedman, S. S. Gubser and A. Karch, "Modeling the fifth dimension with scalars and gravity" Phys. Rev. D 62, 046008 (2000) [arXiv hep-th/9909134]. 
[36] M. R. Garousi, "Tachyon couplings on non-BPS D-branes and Dirac-Born-Infeld action" Nucl. Phys. B 584, 284 (2000) [arXiv:hep-th/0003122.

[37] S. Kachru and L. McAllister, "Bouncing brane cosmologies from warped string compactifications" JHEP 0303, 018 (2003) [arXiv:hep-th/0205209.

[38] A. Kehagias and E. Kiritsis, “Mirage cosmology" JHEP 9911, 022 (1999) [arXiv:hep-th/9910174. 